\title{
Controlled time of arrival windows for already initiated energy-neutral continuous descent operations
}

\author{
Ramon Dalmau and Xavier Prats \\ Technical University of Catalonia - Barcelona Tech, Telecom and Aerospace Engineering \\ School of Castelldefels. Esteve Terradas, 5. Castelldefels, Catalonia (Spain).
}

\begin{abstract}
Continuous descent operations with controlled times of arrival at one or several metering fixes could enable environmentally friendly procedures without compromising terminal airspace capacity. This paper focuses on controlled time of arrival updates once the descent has been already initiated, assessing the feasible controlled time of arrival window (and associated fuel consumption) of continuous descent operations requiring neither thrust nor speedbrake usage along the whole descent (i.e. only elevator control is used to achieve different metering times). Based on previous works, an optimal control problem is formulated and numerically solved. The earliest and latest times of arrival at the initial approach fix have been computed for the descent of an Airbus A320 under different scenarios, considering a wide range of altitudes and distances to go when receiving the controlled time of arrival update. The effects of the aircraft mass, longitudinal wind and position of the initial approach fix on the controlled time of arrival window have also been investigated. Results show that controlled time of arrival windows up to four minutes could be achieved for certain conditions. Interestingly, minimum fuel trajectories almost correspond to the earliest trajectories at the metering fix.
\end{abstract}

Keywords: Air Traffic Management, Trajectory Optimisation, Controlled Time of Arrival, Continuous Descent Operations, Time windows

\section{Introduction}

With the awareness of global warming and the rising of fuel prices, reducing the environmental footprint of aviation has become one of the main 
concerns of the different aviation stakeholders. Continuous descent operations (CDO) have demonstrated and proven success in the reduction of emissions, fuel consumption and noise nuisance in terminal manoeuvring areas (TMA) [5]. In order to get the maximum benefits of CDO, aircraft should descent with the engines at idle from the top of descent (TOD) down to the stabilisation point, where the aircraft is configured and ready for landing (typically $1,000 \mathrm{ft}$ above aerodrome level). Due to the difficulty for air traffic controllers (ATC) to accurately predict the vertical profile of these descent trajectories, larger separation margins are required and in the majority of TMA these procedures are limited to periods of low demand.

An approach to enable CDO without compromising airspace capacity is to assign to each aircraft a controlled time of arrival (CTA) at a metering fix to safely merge incoming traffic [15]. Extensive research has been done in the recent decades on on-board trajectory planning and guidance algorithms capable to fulfil one or various CTA along the descent [27, 20, 9]. Similar efforts have been devoted to improve ground-based CTA assignment algorithms that enable time based sequencing and merging procedures and minimise path stretching instructions by ATC [25, 14, 17].

In order to efficiently allocate these CTA, each inbound aircraft should compute the earliest and latest CTA (CTA window) and report them to the ground systems for proper synchronisation [24]. These CTA windows depend on many factors, including aircraft performance, weather conditions and, noticeably, the aircraft states when receiving the CTA (i.e. altitude, speed and remaining distance to the metering fix).

In this context, several studies have dealt with the assignment of CTA (and the quantification of the feasible CTA windows), when the aircraft is still in cruise, well before the top of descent. See for instance [25, 18, 19] where these windows at a metering fix were computed by allowing the aircraft to adjust the TOD position and the descent speed profile. With similar purposes, Ref. [26] also enabled the addition (resp. omission) of waypoints to stretch (resp. reduce) the flight path length in addition to the TOD adjustment.

In the future trajectory-based air traffic management (ATM) paradigm, we could envisage ATC updating the CTA once the descent has been initiated, and even assigning a CTA to more than one fix along the descent route. In such cases (and aiming at minimising the environmental footprint), aircraft should be capable to keep as much as possible on the CDO without requiring neither additional thrust nor speed-brakes use. This could be done 
by taking advantage of time and energy management [8, 9], which permits to adjust the speed profile by means of elevator control only, keeping the aircraft into the planned lateral route.

The robustness of CDO trajectories in the face of late changes to the CTA during the descent was assessed in [16]. Aiming to minimise the impact on the optimality of the CDO, only elevator control was permitted to adjust the time at which the metering fix was reached, assuming rather simple Mach/calibrated airspeed (CAS) profiles and allowing a single (and instantaneous) modification of the scheduled speed. Furthermore, this assessment was performed for few initial conditions and the employed method could not ensure the optimality (in the mathematical sense) of the trajectories.

Another important limitation of previous works is that none of them (up to our best knowledge) took into account the remaining descent between the concerned metering fix and the runway threshold. Adjusting the speed profile to minimise or maximise flight time may result in a change to the altitude at which the metering fix is overflown. If the energy of the aircraft at this fix were too low, additional thrust would be needed after overflying it (interrupting in this way the CDO) [3]. Similarly, if the energy were too high it would be required to use speed-brakes and/or to deploy high-lifting devices or the landing gear earlier than planned. Furthermore, all previous works used the BADA v3.x performance model, which has been reported to show significant limitations for accurate trajectory prediction in TMA $[23,22,11]$. More sophisticated aerodynamic drag end engine models are needed in order to compute realistic descent profiles and to obtain accurate fuel consumption and flight time figures.

The main contributions of this paper are summarised as follows. Firstly, we assess complete energy-neutral descents (down to the stabilisation point), assuming that the descent has already been initiated when the ATC updates the CTA at a given metering fix. Thus, the earliest and latest times of arrival at this fix are computed for a wide range of initial conditions aiming at quantifying the CTA window as a function of the altitude and distance to go. These trajectories are restricted in such a way that neither thrust nor speed-brakes use is allowed along the whole descent. In this way, the whole trajectory planning is subject to this optimisation where only elevator control is left to modify the speed profile and to adjust the time of arrival of the continuous descent trajectory. Secondly, the CTA window sensitivity to different longitudinal winds, metering fix position and aircraft mass are also investigated. Finally, accurate aircraft performance data derived from 
Airbus Performance Engineering Program (PEP) are used to model drag, engine thrust and fuel flow in order to derive realistic fuel and time figures.

\section{Background}

In the recent years, several research has focused on the use of energy principles to perform accurate, time-constrained, engine-idle descents to reduce the environmental impact of aviation [8, 9, 21]. The idea behind time and energy management is to exchange altitude for speed and vice versa to gain or lose time and energy through elevator control. Following this process, deviations from the plan (including CTA updates) are corrected without the need for additional thrust or speed-brakes use, leading to what is called an energy-neutral trajectory. The earliest and latest energy-neutral trajectories at a metering fix can be computed by solving a trajectory optimisation problem, where the time of arrival at the fix is minimised or maximised at the same time different operational constraints are satisfied (and namely the impossibility to use thrust or speed-brakes during the descent).

Trajectory optimisation requires the definition of a mathematical model describing the aircraft dynamics along with a model for certain atmospheric variables. Section 2.1 shows these equations of motion, while Section 2.2 provides a detailed description of the time and energy management concept. Finally, in Section 2.3 the formulation of the optimisation problem for an aircraft trajectory is presented.

\subsection{Aircraft point-mass model}

In this paper, the dynamics of the aircraft are expressed by the following set of non-linear ordinary differential equations (ODE), assuming a pointmass representation of the aircraft reduced to what is commonly called a gamma-command model (where continuous vertical equilibrium is assumed) and neglecting the vertical and crosswind components:

$$
\begin{aligned}
& \frac{\mathrm{d} v}{\mathrm{~d} t}=\dot{v}=\frac{T_{\text {idle }}-D}{m}-g \sin \gamma \\
& \frac{\mathrm{d} s}{\mathrm{~d} t}=\dot{s}=v \cos \gamma+W_{s} \\
& \frac{\mathrm{d} h}{\mathrm{~d} t}=\dot{h}=v \sin \gamma
\end{aligned}
$$


where the state vector $\boldsymbol{x}=[v, s, h]$ is composed respectively, by the true airspeed (TAS), the distance to go and the altitude of the aircraft; $T_{i d l e}$ is the idle thrust; $D$ is the aerodynamic drag; $W_{s}$ is the longitudinal wind (headwind or tailwind); and $g$ is the gravity acceleration. Since neither additional thrust nor speed-brakes use is permitted, the control vector is composed by the aerodynamic flight path angle only (i.e. $\boldsymbol{u}=[\gamma]$ ). Note that the variations in mass $m$ are neglected in the dynamic model because the fuel consumption during an idle descent is a very small fraction of the total mass [5]. In spite of that, the fuel flow idle $F F_{\text {idle }}$ is computed in the model to determine the amount of fuel consumption for results assessment.

All aerodynamic and engine parameters (needed to model $T_{\text {idle }}, D$ and $\left.F F_{\text {idle }}\right)$ have been modelled by approximating the manufacturers tabular performance data by tensor product cubic B-splines, as detailed in Section 2.2.

Regarding the atmosphere, the International Standard Atmosphere [12] model is considered, which defines the density $\rho$, pressure $p$ and temperature $\tau$ magnitudes as functions of $h$. The longitudinal wind is assumed to be constant at high altitudes down to a reference height $h_{r}$. Below $h_{r}$ the well known Hellmann exponential law is used [10]:

$$
W_{s}= \begin{cases}W_{r} & \text { if } h>h_{r} \\ W_{r}\left(\frac{h}{h_{r}}\right)^{\alpha} & \text { if } h \leq h_{r}\end{cases}
$$

where $W_{r}$ is a known wind speed at $h_{r}$. The Hellman exponent $(\alpha)$ depends on many factors, such as the coastal location, the shape of the terrain on the ground, and the stability of the air. In this paper $\alpha=1 / 7$ has been considered, which is the typical value for neutral stability conditions [13].

Finally, it should be noted that operational constraints are usually given in terms of the Mach number $M$ or CAS $v_{C A S}$, which both can be computed as a function of the TAS and the atmospheric magnitudes.

\subsection{Time and energy management}

The total energy $E_{t}$ of an aircraft is the sum of its kinetic energy $E_{k}$ and potential energy $E_{p}$ :

$$
E_{t}=E_{k}+E_{p}=\frac{1}{2} m v^{2}+m g h .
$$

The energy rate can be obtained by differentiating Eq. (3): 


$$
\dot{E}_{t}=m v \dot{v}+m g \dot{h} .
$$

By combining Eqs. (4) and (1) the total energy rate in a CDO can be expressed in terms of the forces acting upon the aircraft:

$$
\dot{E}_{t}(h, v, \gamma)=v\left(T_{i d l e}-D\right) .
$$

According to Eq. (5) the total energy of an aircraft could be increased by applying thrust and decreased by increasing drag. However, during a CDO aircraft should fly at idle thrust and speed-brakes use should be avoided. In such conditions, the energy rate can be (indirectly) controlled by adjusting the altitude and speed profiles due to their impact on the idle thrust and drag.

Aiming to reflect these dependences, a performance model for an Airbus A320 has been developed using accurate data from the manufacturer. Typical aerodynamic and engine data are specified in tabular form, generally obtained as a result of experimental tests. For optimisation solvers to work efficiently, continuity and differentiability for the right hand sides of the model equations are required. Although some of the performance data could be well approximated by polynomial functions, this method is prone to oscillation due to the Runge's phenomenon, leading to poor convergence or local minima issues.

In this paper, twice-differentiability is achieved by approximating the performance data by tensor product cubic B-splines as suggested in Ref. [2]. The aerodynamic drag is modelled as:

$$
D=\frac{1}{2} \rho S v^{2} C_{D},
$$

where $C_{D}$ is the drag coefficient and $S$ is the wing area. Aerodynamic data from the manufacturer has been taken to approximate $C_{D}$ as a function of the lift coefficient $C_{L}$, the Mach number $M$ (which in turns depends on $h$ and $v$ ) and $h$ by a tensor product cubic B-spline of the form:

$$
C_{D}\left(C_{L}, M, h\right)=\sum_{i, j, k} c_{i, j, k} B_{i}\left(C_{L}\right) B_{j}(M) B_{k}(h),
$$

where $B_{l}$ (with $l \in\{i, j, k\}$ ) are B-spline basis functions and $c_{i, j, k}$ are the B-spline control points [7]. $C_{L}$ is obtained by assuming continuous vertical equilibrium: 


$$
C_{L}=\frac{2 m g \cos \gamma}{S \rho v^{2}}
$$

Following the same methodology, $T_{i d l e}$ and $F F_{\text {idle }}$ are expressed as a function of $h$ and $M$ :

$$
\begin{aligned}
T_{\text {idle }}(h, M) & =\sum_{i, j} c_{i, j} B_{i}(h) B_{j}(M), \\
F F_{\text {idle }}(h, M) & =\sum_{i, j} c_{i, j} B_{i}(h) B_{j}(M) .
\end{aligned}
$$

Eq. (9) is valid for all the descent down to the stabilisation point (typically at $1000 \mathrm{ft}$ ). For the remainder of the descent, the aircraft thrust $T$ is left free between $T_{\text {idle }}$ and the maximum thurst $T_{\max }$ and the nominal fuel flow $F F$ is computed as a function of $T, M$ and $h$. Both $T_{\max }$ and $F F$ are also modelled by approximating the manunfacturer data with tensor product cubic B-splines.

Finally, it should be noted that the law of conservation of energy states that potential energy can be exchanged for kinetic energy and vice versa. Therefore, even if not permitting neither the use of speed-brakes nor additional thrust, it is still possible to adjust the altitude and speed profiles by using the elevator control only. Namely, if the aircraft requires a higher velocity, it could loose altitude instead of applying additional thrust. Alternatively, it could reduce speed by pitching up instead of using drag devices.

\subsection{Optimal control problem formulation}

The optimisation of an aircraft trajectory can be formulated as a multiphase constrained optimal control problem, in which it is desired to determine the controls of a system such that a given cost function $J$ is maximised or minimised while satisfying a set of constraints. For some simple problems, the solution can be obtained analytically from the necessary and sufficient conditions of optimality [4]. However, finding the analytical solution when strong non-linear functions or interior point constraints appear in the definition of the model (as in the problem discussed herein) is not a straightforward task. In such cases, it is necessary to employ numerical methods [2].

Numerical methods for solving optimal control problems can be divided into two major classes: indirect methods and direct methods. In this paper, 
the latter have been used because they can easily cope with inequality constraints, among many other advantages [2]. Such direct methods transform the original continuous (and thus infinite) optimal control problem into a (discrete and finite) non-linear programming (NLP) optimisation problem. The time histories of control and state variables are discretised at a set of collocation points, being the system of ODE of Eq. (1) approximated by some continuous function (such as polynomials) over each collocation step. The values of these discretised variables, along with some (optional) nontime dependent parameters, become the unknowns of the new finite variable problem, which can be formally as a NLP problem and solved by standard NLP solvers.

Several collocation schemes are proposed in the literature, being the trapezoidal collocation method the approach used in this paper. Trapezoidal collocation shows a good trade-off between accuracy and execution time needed to solve highly constrained NLP problems [2]. Further mathematical details on the formulation of optimal control problems for the optimisation of aircraft trajectories can be found in Ref. [6].

\section{Earliest and Latest trajectory computation}

The optimisation process presented in this paper is a constrained nonlinear optimal control problem, as described in Section 2.3. The study presented here aims at computing minimum and maximum time trajectories at a given metering fix, which is assumed to be at the initial approach fix (IAF) of an hypothetical approach procedure. Accordingly, the cost function is defined as:

$$
J=t_{\mathrm{IAF}}
$$

where $t_{\mathrm{IAF}}$ is the time of arrival at the IAF. Constraints on the aircraft dynamics are particularised by the point-mass model given by Eq. (1); the box constraint on the control variable is specified as:

$$
\gamma_{\min } \leqslant \gamma \leqslant 0
$$

where $\gamma_{\min }$ is the minimum flight path angle, chosen according to aircraft operational limitations; and generic path constraints, defined as functions of the state variables, are also specified to ensure that the aircraft speed remain within operational bounds: 


$$
\text { FAS } \leqslant v_{C A S} \leqslant \mathrm{VMO} ; M \leqslant \text { MMO. }
$$

FAS is the final approach speed; VMO and MMO are, respectively, the maximum operational $v_{C A S}$ and $M$.

The descent is split in different phases, where different phase-dependent path and event constraints may apply, in addition to previous ones. Table 1 shows the different phases and their associated extra constraints (note that in this table $t_{0}$ and $t_{f}$ refers, respectively, to the initial and final time of the concerned phase).

Table 1: Definition of the flight phases and their constraints

\begin{tabular}{lcc}
\hline Phase & Path Constraints & Event constraints \\
\hline Descent above FL100 & $\mathrm{GD} \leq v_{C A S}(t)$ & $h\left(t_{0}\right)=h_{0} ; s\left(t_{0}\right)=s_{0}$ \\
Descent below FL100 & $\mathrm{GD} \leq v_{C A S}(t) \leq 250 \mathrm{kt}$ & $h\left(t_{0}\right)=10000 \mathrm{ft}$ \\
Approach & $\mathrm{GD} \leq v_{C A S}(t) \leq 250 \mathrm{kt}$ & $s\left(t_{0}\right)=s_{\mathrm{IAF}}$ \\
Leveled Deceleration & $h(t)=2000 \mathrm{ft} ; \mathrm{S} \leq v_{C A S}(t)$ & $v_{C A S}\left(t_{0}\right)=\mathrm{GD} ; s\left(t_{0}\right)=s_{\mathrm{FAP}}-2 \mathrm{NM}$ \\
Deceleration on glide path & $\gamma(t)=-3^{\circ}$ & $v_{C A S}\left(t_{0}\right)=\mathrm{S} ; s\left(t_{0}\right)=s_{\mathrm{FAP}}$ \\
Stabilised on glide path & $\gamma(t)=-3^{\circ} ; v_{C A S}(t)=\mathrm{FAS}$ & $h\left(t_{0}\right)=1000 \mathrm{ft} ; h\left(t_{f}\right)=50 \mathrm{ft} ; s\left(t_{f}\right)=0$ \\
\hline
\end{tabular}

${ }^{*}$ Additional thrust is allowed in this particular phase in order to maintain the final approach speed while keeping on the ILS glide path.

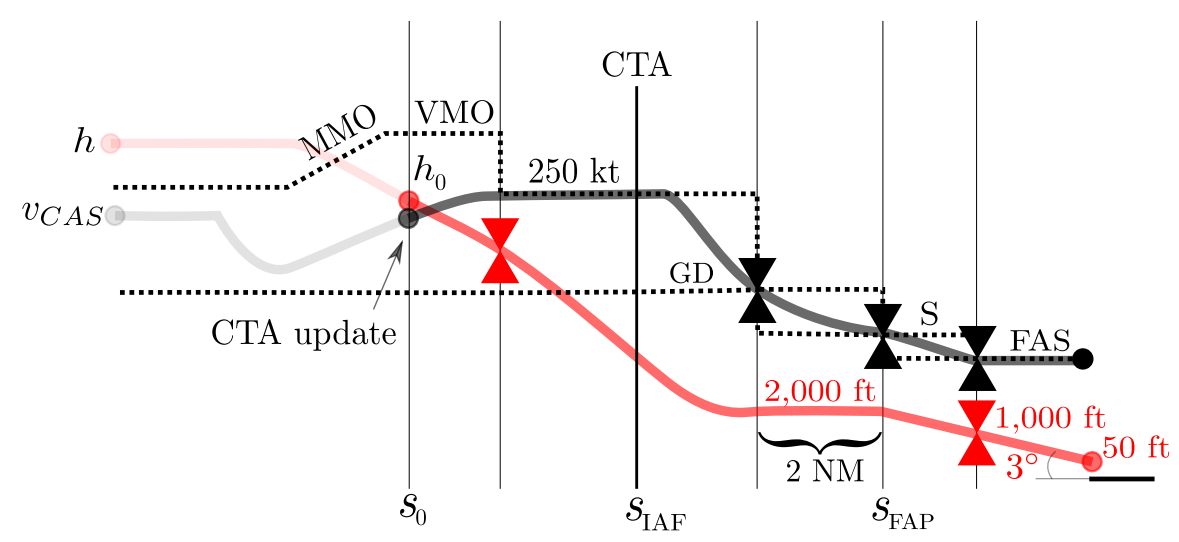

The first phase starts at the initial conditions (defined by $h_{0}$ and $s_{0}$ ) and ends at FL100. Below this altitude, ATC procedures typically restrict the CAS to $250 \mathrm{kt}$. Afterwards, the aircraft directs to the IAF, where the approach phase begins. Two nautical miles before the final approach point 


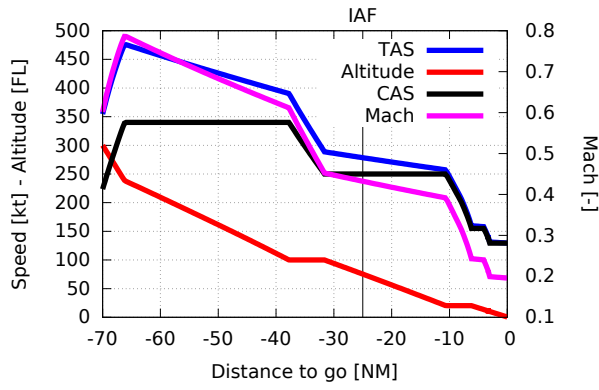

(a) High $E_{p}$ earliest trajectory

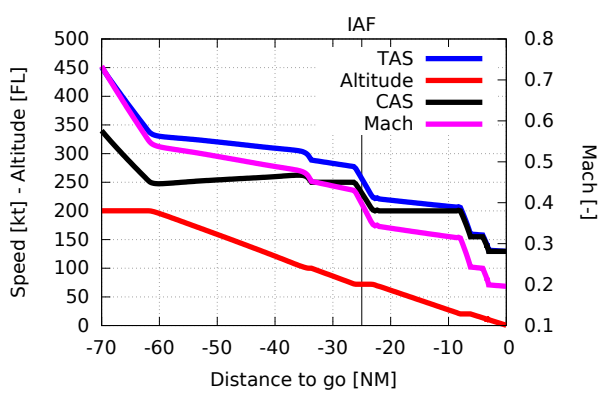

(c) Low $E_{p}$ earliest trajectory

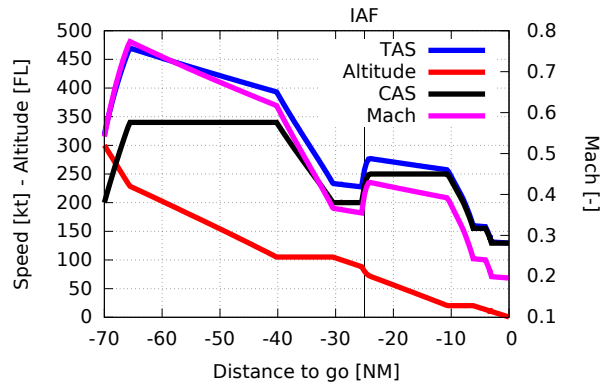

(b) High $E_{p}$ latest trajectory



(d) Low $E_{p}$ latest trajectory

Figure 1: Examples of energy-neutral trajectories

(FAP), the aircraft decelerates to green dot speed GD and starts configuring with flaps at constant altitude (still at idle thrust), in order to intercept the instrumental landing system (ILS) glide path at the FAP and at the S speed.

When descending on the ILS glide path, the aircraft decelerates at idle thrust and reaches the FAS at $1000 \mathrm{ft}$ with the gear down and in landing configuration. The last phase terminates upon the aircraft reaching $50 \mathrm{ft}$ over the runway threshold at the FAS. It should be noted that nominal flap/slat (and landing gear) transitions are also considered as additional trajectory phases, but not depicted in Table 1 aiming to keep it simple.

Event constraints for the state variables fix the initial and final conditions of the problem. In this paper, only the initial altitude and distance to go are enforced. Consequently, the initial airspeed (and therefore energy) is left free, assuming that the transition from any airspeed to that chosen by the optimiser would be quick enough in practice by using speed on elevator. The final states of the optimisation problem are fixed at the moment the aircraft reaches $50 \mathrm{ft}$ over the runway threshold at the FAS. 
Figure 1 shows four examples of optimal trajectories computed with the aforementioned model for an Airbus A320 located at 70 NM from the runway threshold.

Figure 1(a) and 1(b) show, respectively, the earliest and latest trajectories at the IAF for a situation where the aircraft has excess of potential energy. In both cases, the trajectory aims to increase the aerodynamic drag in order to release enough potential energy so that all the constraints are satisfied. Initially, altitude is exchanged by speed at the maximum descent gradient until VMO is reached. Thereafter, the descent is performed at this speed down to FL100, where the aircraft levels-off (but maintaining thrust idle) in order to decelerate as quick as possible. Below this altitude, the earliest and latest trajectories keep, respectively, the maximum and minimum allowed CAS until the IAF position is reached (depicted in Fig. 1 as a vertical black line). What happens after the IAF has no impact on the cost function. Yet, all the constraints in Table 1 must still be satisfied and it does impact on the shape of the whole trajectory (including those phases before the IAF).

By contrast, Figs. 1(c) and 1(d) show, respectively, the earliest and latest trajectories for a situation where the aircraft lacks from potential energy. For the earliest trajectory, it starts at the maximum allowed speed in order to keep this initial energy as much as possible for the remainder of the descent. The latest trajectory consists of an initial speed corresponding to the minimum energy needed to satisfy all the constraints and follows by gliding at GD.

\section{Numerical results}

This section presents the results obtained after computing the earliest and latest trajectories at the IAF for different conditions. First, the experimental design matrix is presented, defining the independent variables considered in the study and enumerating the different simulations. Then, the results for

a baseline scenario are shown an discussed in detail. Finally, the effects of the independent variables considered in the study on the CTA window are investigated.

\subsection{Experiment design matrix}

The following generic scenario has been considered in this paper: an aircraft attempts to perform an energy-neutral CDO and somewhere in the descent (before reaching the IAF) the ATC requests (or updates) a CTA at 
Table 2: Design matrix for the sensitivity study

\begin{tabular}{lccc}
\hline Independent variable & Negative variation & Baseline scenario & Positive variation \\
\hline$m[\% \mathrm{MLM}]$ & 80 & 90 & 100 \\
$s_{\mathrm{IAF}}[\mathrm{NM}]$ & 15 & 25 & 50 \\
$W_{s}[\mathrm{kt}]$ & $-60,-40,-20$ & 0 & $20,40,60$ \\
\hline
\end{tabular}

the IAF. The goal for the study is to quantify the feasible CTA window at this metering fix (latest arrival time - earliest arrival time) as a function of the aircraft altitude and distance to go when receiving the ATC request.

For a given aircraft type and assuming the descent profile presented in Table 1, the earliest and latest times of arrival could be affected by the aircraft mass, the longitudinal wind and the exact location of the IAF along the arrival route. In this paper, a scenario with typical values for those parameters is taken as baseline. For each independent variable, negative and positive variations are considered, aiming to investigate the CTA window sensitivity. A total of 11 representative scenarios have been chosen for this sensitivity study, which are summarised in Table 2.

For all the scenarios shown in Table 2, the minimum gradient $\gamma_{\min }$ has been set to $-15^{\circ}$ and $s_{\mathrm{FAP}}$ has been fixed at $6 \mathrm{NM}$. For each scenario, the green dot speed has been computed according to Ref. [1]. Regarding the characteristic speeds, both S and FAS have been computed as a fraction of the stall speed, which is a function of both aircraft mass and aerodynamic configuration. Finally, the maximum operational speeds MMO and VMO have been set to 0.80 and $340 \mathrm{kt}$, respectively [1].

For each scenario, the earliest and latest trajectories at the IAF have been computed starting at several initial conditions (corresponding to the moment at which the CTA update is notified) and enforcing an energy-neutral descent (neither thrust nor speed-brakes allowed). Initial altitudes between FL100 and FL360 and distances to go ranging from $s_{\mathrm{IAF}}+5 \mathrm{NM}$ to $120 \mathrm{NM}$ have been considered in this study.

It should be noted that, for each initial condition, there exist many energy-neutral trajectories while satisfying all the constraints. Only one of them, however, is optimal in terms of fuel consumption. In order to quantify the extra fuel consumption that earliest and latest trajectories would entail, the minimum fuel trajectory has been also computed for each initial condition of the baseline scenario. 
Results have been obtained using CONOPT as NLP solver, bundled into the GAMS software suite.

\subsection{Baseline scenario results}

This section thoroughly discuses the results obtained for the baseline scenario, whose independent variables were presented in Table 2. Figs. 2(a) and 2(b) show, respectively, the earliest and latest times of arrival at the IAF as a function of the initial altitude and distance to go of the aircraft. The black dashed line represents the trajectory that maximises the CTA window (i.e. the difference between earliest and latest times of arrival at the metering fix) throughout the descent. An aircraft starting the descent in the white region would need to apply additional thrust or to deploy speed-brakes to satisfy all the constraints.

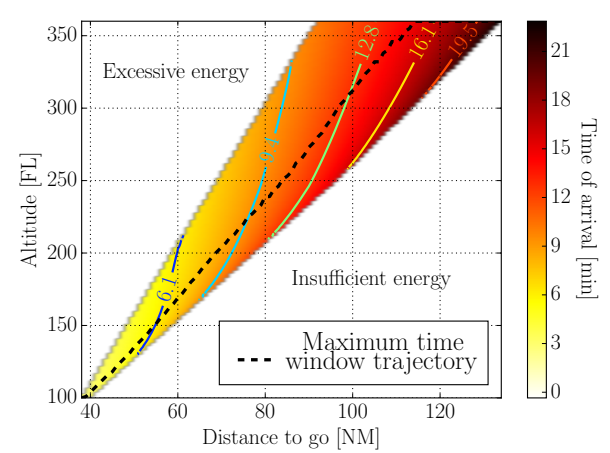

(a) Earliest CTAs

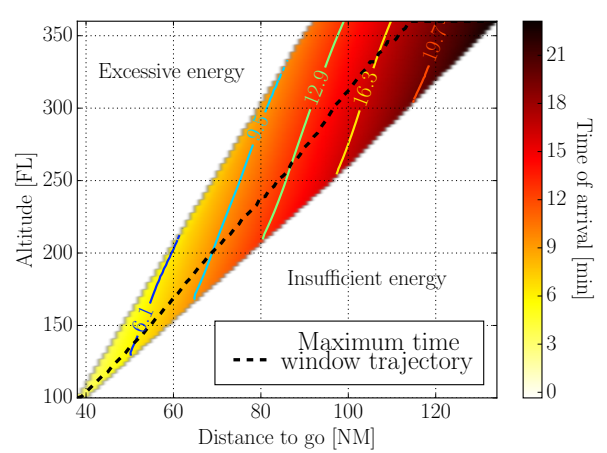

(b) Latest CTAs

Figure 2: Baseline scenario: Earliest and latest CTAs at the IAF for energy neutral CDO

As expected, for a given altitude, the closer (resp. farthest) to the metering fix, the sooner (resp. later) the aircraft would be able to reach it. For a given distance, at the highest feasible altitudes the aerodynamic drag needs to be increased in order to release potential energy. Since speed-brakes use is not allowed, the best option is to accelerate, thus penalising the latest trajectories (see Fig. 1(b)).

As the altitude decreases, the potential energy needed to be released in order to satisfy the trajectory constraints is also reduced. At a given altitude, no acceleration is longer required, resulting in the maximum achievable latest time. Below this altitude, the initial kinetic energy needs to be increased in order to have enough total energy to reach the runway threshold at the 
required altitude and airspeed. In such case, the higher initial speed penalises the latest trajectory, as shown in Fig. 1(d).

Figure 3 shows the CTA window at the IAF for energy-neutral CDO, as a function of the aircraft position when the CTA is updated during the descent.

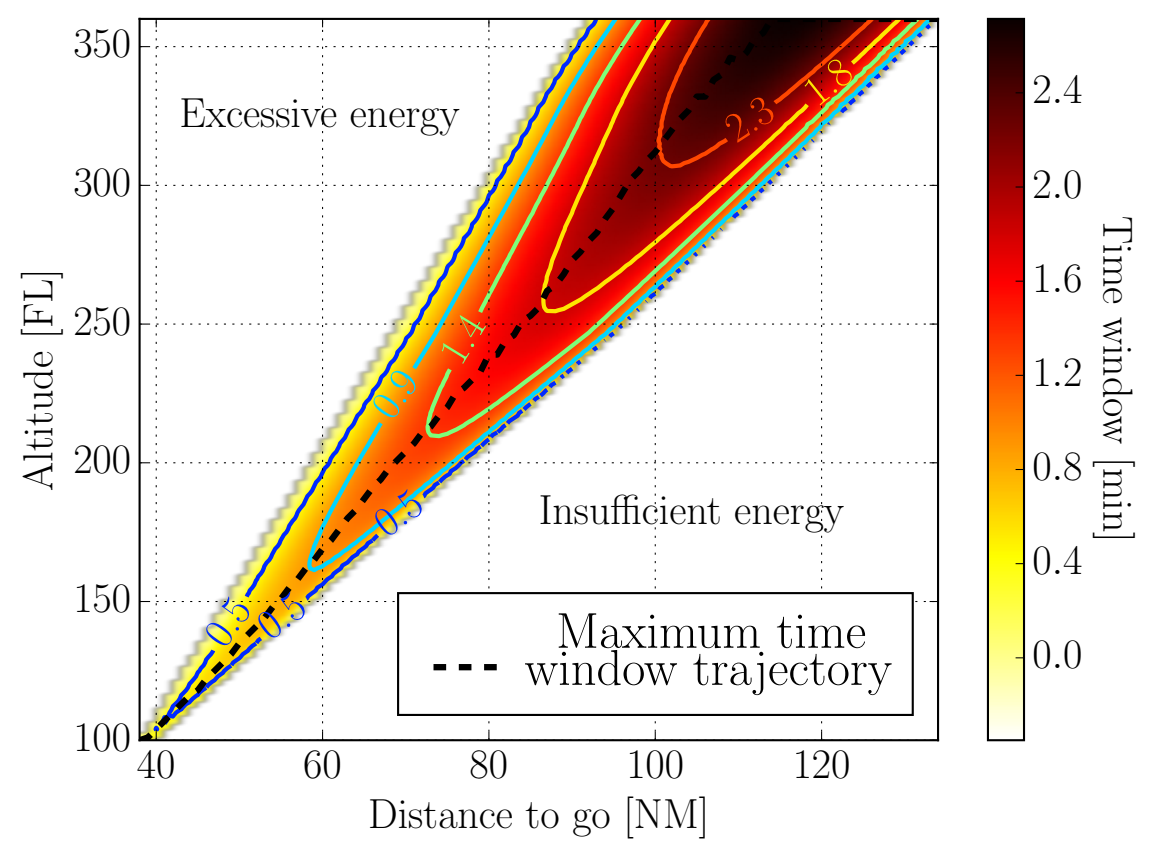

Figure 3: Baseline scenario: CTA window at the IAF for energy neutral CDO

According to Figs. 2 and 3, there exist only a feasible region of initial distances and altitudes such that an aircraft could satisfy all the constraints without requiring neither additional thrust nor drag devices. If the aircraft is close to the lower border of this feasible region, the potential energy needs to be sustained by means of a shallower flight path angle and almost no margin is left to increase the aircraft speed using elevator control. As the aircraft position approaches the trajectory of maximum CTA window, it has more freedom either to accelerate or decelerate by means of elevator control and the CTA window increases until it reaches its maximum. However, if the aircraft is close to the upper border of the feasible region, potential energy needs to be exchanged by kinetic energy by descending with a steeper gradient and 
almost no margin is left to decrease the aircraft airspeed.

For this baseline scenario, the maximum time window (approximately $4 \mathrm{~min}$ ) is reached at the higher altitude (FL360) and at $114 \mathrm{NM}$. It is reduced almost $2.4 \mathrm{~s}$ per NM or $7.8 \mathrm{~s}$ per $1000 \mathrm{ft}$.

Another conclusion that arises from Fig. 3 is that the CTA window strongly depends on the position of the TOD, which in turn is a function of many factors such as the cost index (CI), the aircraft mass, the scheduled speed profile, the wind field or even a previous CTA assigned in cruise. For instance, depending on the CI values the location of the TOD will be "moved" towards or away the metering fix. Too high or too low CI values will narrow the CTA window while intermediate CI values will place the TOD in such a way that a wider window could be achieved.

The goal of energy-neutral trajectories is to correct deviations from the plan (including CTA updates) with the minimum impact on the optimality of the operation. Nevertheless, even if keeping the engines at idle throughout the descent, the resulting fuel consumption depends on the aircraft altitude and (to a lesser extent) speed profiles. Figs. 4(a) and 4(b) show, respectively, the fuel consumption of the earliest and latest trajectories at the IAF, as a function of the initial position of the aircraft. As expected, the total fuel consumption and the time of arrival at the metering fix are strongly correlated.

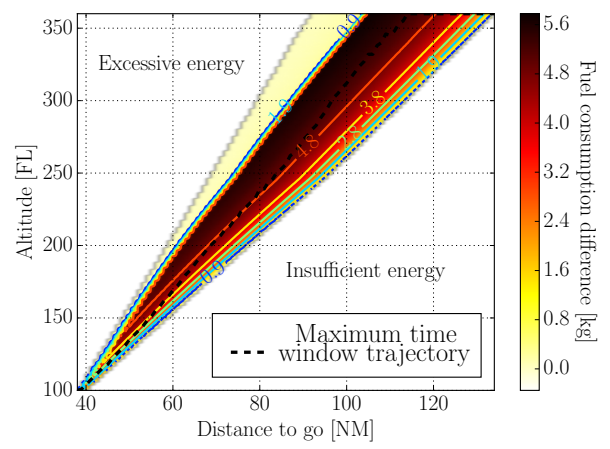

(a) Earliest CTA trajectories

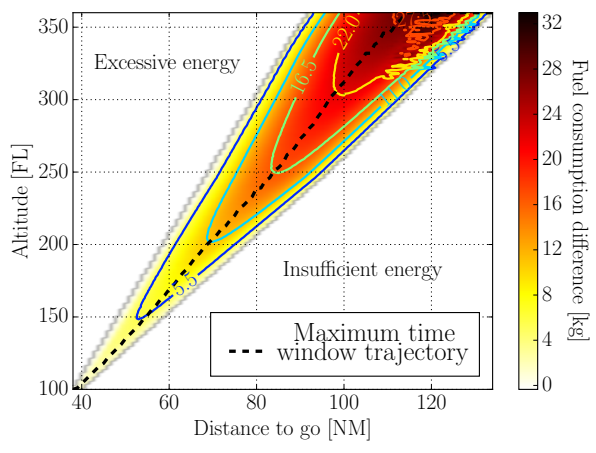

(b) Latest CTA trajectories

Figure 4: Baseline scenario: Fuel consumption at the IAF for energy-neutral CDO

In Fig. 4 significant differences in fuel consumption, between the earliest and latest trajectories, are observed, even though both are performed with 
the engines at idle. Interestingly, it can be observed that earliest trajectories are more fuel-efficient than latest trajectories.

A priori, this could be seen as a paradox, since one might think that the higher the aircraft speed, the higher the fuel consumption. If the CTA were updated in cruise, earliest trajectories would select a later TOD, leading to higher fuel consumption due to the larger amount of time spent in the cruise phase. In the scenario discussed in this paper, however, the aircraft is already descending when the CTA is updated and only elevator control is allowed to gain or loose time. The idle fuel flow depends mainly on altitude, being lower at high altitudes. The latest trajectories tend to release as much energy as possible at the beginning of the descent, then flying at low speeds and low altitudes. On the other hand, earliest trajectories attempt to keep the faster speeds at the higher altitudes where the density (and the drag) are lower in order to maintain the total energy level as long as possible, then releasing this energy close to the constrained metering fix. The more fuelefficient altitudes, at which earliest trajectories operate, result in lower fuel consumption.

Among all the possible energy-neutral trajectories, there exist only one that minimises fuel consumption for each initial condition. Figs. 5(a) and 5(b) show, respectively, the extra fuel consumption of the earliest and latest trajectories at the IAF with respect to the minimum fuel trajectory.

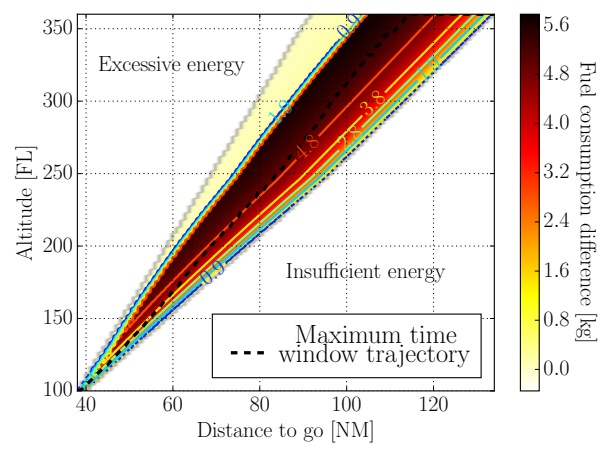

(a) Earliest CTA trajectories

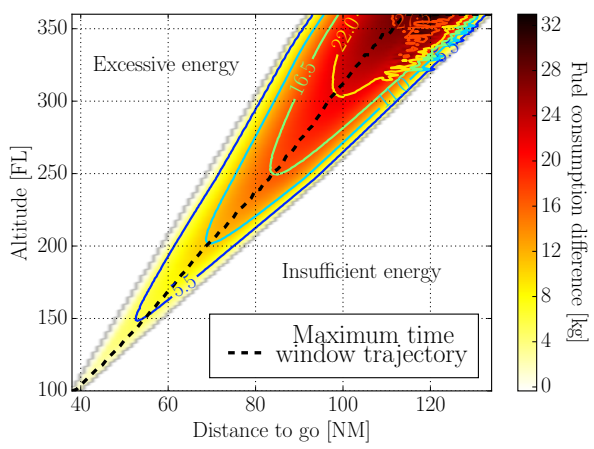

(b) Latest CTA trajectories

Figure 5: Extra fuel consumption with respect to the minimum fuel trajectory at the IAF

According to Fig. 5(a) the most fuel-efficient trajectories at the IAF are very close to those of minimum time, since both select similar altitude and speed profiles. Interestingly, the trajectory that maximises the CTA window 
throughout the descent also entails the maximum extra fuel consumption with respect to the minimum fuel trajectory for the same initial conditions. Consequently, there is a trade-off between maximising the CTA window (and therefore maximising the probability to achieve the whole descent at thrust idle) and minimising the fuel consumption.

\subsection{Sensitivity study results}

This Section sums up the main results obtained from the sensitivity study. Results have been classified according to the independent variable subject of analysis.

\subsubsection{Effect of aircraft mass}

Figure 6 shows the effect of aircraft mass on the CTA window, where it can be seen that the mass has little influence on the feasible set of initial positions at which an aircraft could achieve an energy-neutral CDO. However, the CTA window for each one of these positions strongly depends on the aircraft mass, being wider for lower masses.
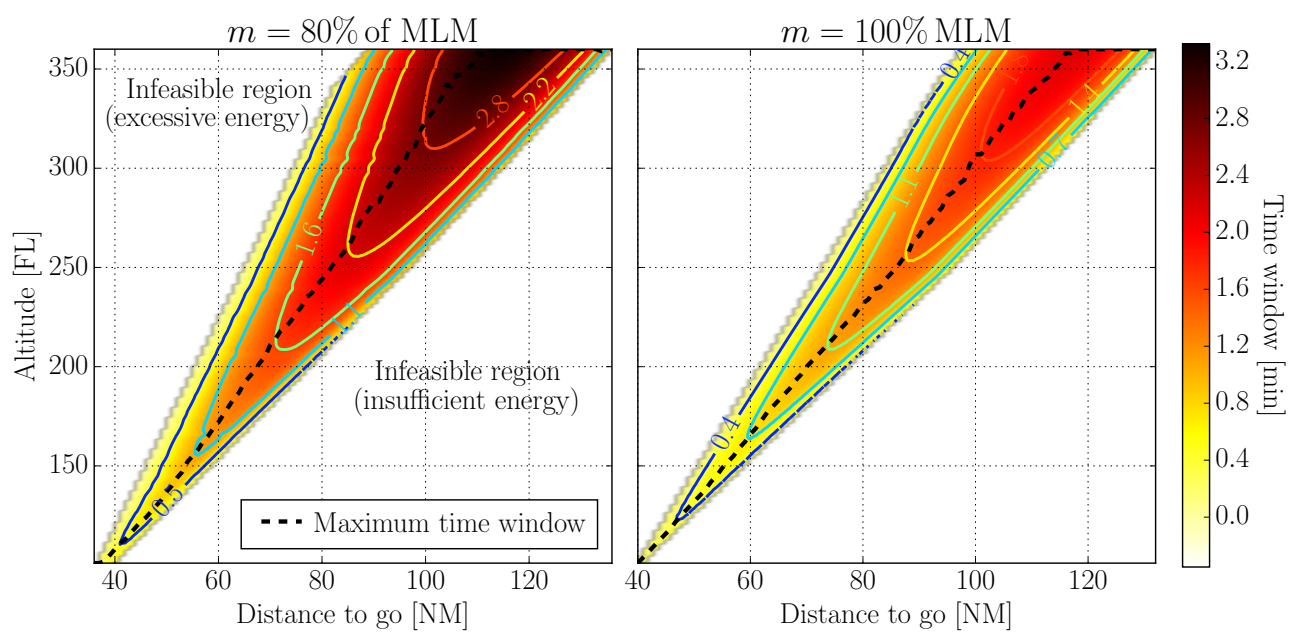

Figure 6: Effects of aircraft mass on the CTA window for energy-neutral CDO

The best glide speed of an aircraft is found at the maximum Lift-toDrag ratio, which only depends on the aerodynamic characteristics of the aircraft. However, the speed at which the maximum Lift-to-Drag ratio occurs 
(approximately GD) is a function of the aircraft mass. For a given altitude, the heavier the aircraft, the higher the GD speed.

When the involved aircraft is in clean configuration, fully automated and flying the trajectory as determined by the flight management system (FMS), the CAS is not allowed to drop below GD [1]. Therefore, while the earliest times of arrival keep almost unchanged with the aircraft mass (since VMO is independent of $m$ ), slower descents can be achieved with lighter weights because of the lower GD speeds. Consequently, there is a trade-off between maximising the CTA window and maximising the amount of payload.

It is worth noting that the difference of CTA window between two different aircraft masses is not position-independent, but becomes more significant at farthest distances from the runway threshold. For instance, for an increment of the aircraft mass from $80 \%$ to $100 \%$ of MLM, at 60 NM from the runway threshold the maximum CTA window decreases one minute, while at $110 \mathrm{NM}$ the detriment is about 2.5 minutes.

\subsubsection{Effect of longitudinal wind}

Figure 7 shows the effect of the longitudinal wind on the CTA window in a matrix form. Each column represents a different magnitude of the wind speed. The first row shows the results for the simulations with head wind, while the second row for those with tail wind.
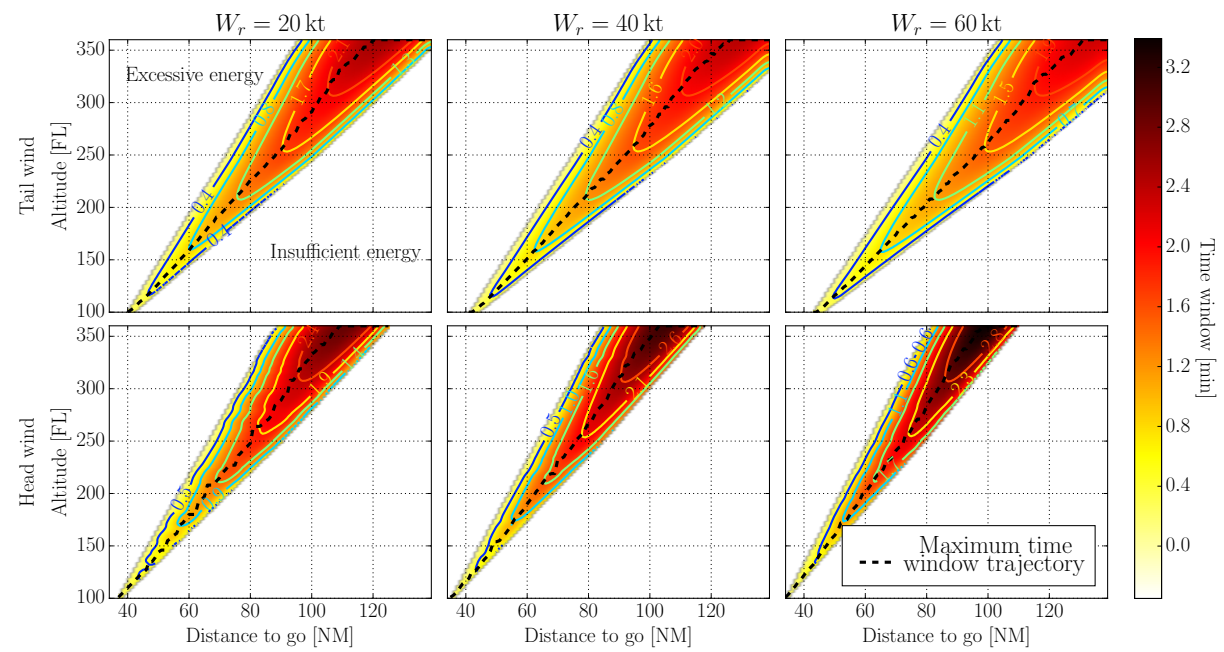

Figure 7: Effects of longitudinal wind on the CTA window for energy-neutral CDO 
According to Fig. 7, in presence of head wind the feasible range of initial positions "moves" towards the metering fix and becomes narrower, but the achievable CTA window increases, if compared with calm wind conditions (see Fig. 3). The opposite behaviour applies in presence of tail wind.

This observation is easier to understand by analysing the impact of the longitudinal wind on the total energy lost per unit length, which expression can be obtained by combining Eqs. (1) and (5):

$$
\frac{\mathrm{d} E_{t}}{\mathrm{~d} s}=\frac{\mathrm{d} E_{t}}{\mathrm{~d} t} \frac{\mathrm{d} t}{\mathrm{~d} s}=\frac{\dot{E}_{t}}{\dot{s}}=\frac{v\left(T_{\text {idle }}-D\right)}{v \cos \gamma+W_{s}} .
$$

Equation (13) leads to the following inequalities for a given weight, altitude and speed:

$$
\left.\frac{\mathrm{d} E_{t}}{\mathrm{~d} s}\right|_{W_{s}>0}<\left.\frac{\mathrm{d} E_{t}}{\mathrm{~d} s}\right|_{W_{s}=0}<\left.\frac{\mathrm{d} E_{t}}{\mathrm{~d} s}\right|_{W_{s}<0} .
$$

According to Eq. (14), in presence of head (resp. tail) wind, less (resp. more) distance is needed to release the same amount of energy if compared with calm winds condition. Namely, for a given initial $E_{t}$, an aircraft flying in presence of head wind would need to start the descent closer to the metering fix to fulfill all the constraints. Therefore, Eq. (14) explains why, for a given altitude, the set of initial positions "moves" towards the metering fix and becomes narrower for the head wind scenarios.

Ideally, for the earliest trajectories it would be desirable to maintain the maximum allowed speed (either MMO/VMO or $250 \mathrm{kt}$ depending on the altitude) until the metering fix. Similarly, for the latest trajectories the deceleration to GD should be performed as soon as possible. In practice, however, the capability to release energy once the IAF is overflown constrains the altitude and speed at which this fix can be reached, and the ideal conditions stated above cannot always be met.

For the earliest scenarios in presence of head wind, the aircraft reaches FL100 at VMO and decelerates to $250 \mathrm{kt}$ just before the IAF, since it is capable of releasing such amount of energy in the remaining distance towards the runway threshold $\left(s_{I A F}\right)$. In presence of tail wind, however, the energy at the IAF cannot be as high because of the lower $\left|\mathrm{d} E_{t} / \mathrm{d} s\right|$, and the deceleration to $250 \mathrm{kt}$ must be performed well before the metering fix. 


\subsubsection{Effect of IAF position}

Figure 8 shows the effect of the position of the metering fix on the CTA window. As expected, the closer the metering fix to the runway threshold, the wider the CTA window. It is interesting to note that, even if the CTA window is significantly affected by the position of the metering fix, the set of feasible initial positions is the same. This is because of the particularities of the selected scenario, in which neither speed nor altitude constraints have been considered. As a result, the set of feasible solutions is not affected by the IAF location, but only the value of the cost function $\left(t_{I A F}\right)$. It is expected that, by considering more realistic scenarios with speed and/or altitude constraints at the IAF, not only $t_{I A F}$ but also the set of feasible initial positions would be affected.
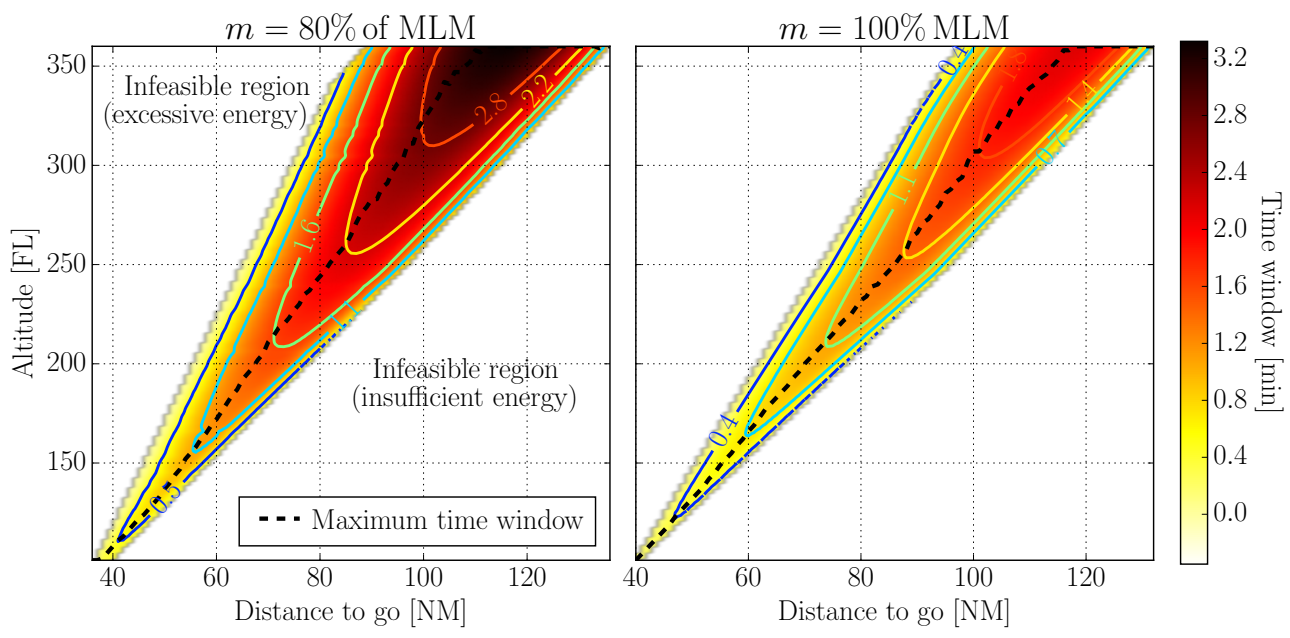

Figure 8: Effects of $s_{\text {IAF }}$ on the CTA window for energy-neutral CDO

\subsubsection{Effect of initial energy}

Figure 8 shows the effect of the position of the metering fix on the CTA window. As expected, the closer the metering fix to the runway threshold, the wider the CTA window. It is interesting to note that, even if the CTA window is significantly affected by the position of the metering fix, the set of feasible initial positions is the same. This is because of the particularities of the selected scenario, in which neither speed nor altitude constraints have been considered. As a result, the set of feasible solutions is not affected 
by the IAF location, but only the value of the cost function $\left(t_{I A F}\right)$. It is expected that, by considering more realistic scenarios with speed and/or altitude constraints at the IAF, not only $t_{I A F}$ but also the set of feasible initial positions would be affected.
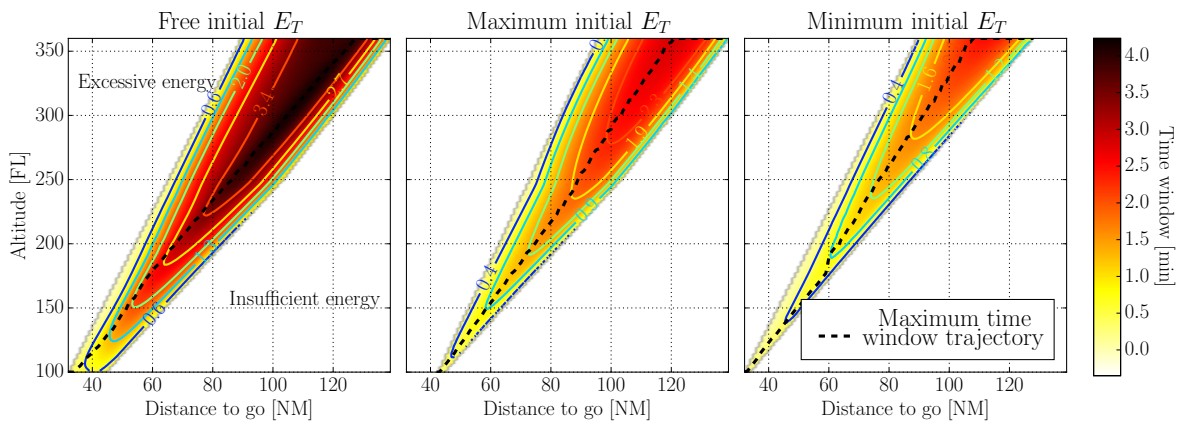

Figure 9: Effects of the initial energy on the CTA window for energy-neutral CDO

\section{Conclusion}

An efficient assignment of controlled times of arrival (CTA) when sequencing and merging traffic could enable the introduction of continuous descent operations (CDO) in the terminal airspace, where conflictive trade-offs exist between airspace capacity and environmental objectives. For this purpose, future ground based-systems will require a good knowledge of the feasible CTA windows for each inbound aircraft. This paper quantified this window at the initial approach fix (IAF) for an Airbus A320 performing a CDO such that the speed profile is only adjusted by means of elevator control along the whole descent and assuming the descent has been already initiated.

Results for a baseline scenario show that CTA windows up to $4 \mathrm{~min}$ at the IAF could be achieved for certain initial conditions. Another important remark that arises from this study is that a previously assigned CTA or even the cost index could have a significant impact on the achievable CTA window.

If has been also found that the lighter the aircraft, the wider the CTA window for the same conditions. In addition, in presence of tail wind, the feasible range of initial conditions such that an energy-neutral trajectory can be applied, narrows and "moves" towards the metering fix, but the CTA window increases. Finally, and as expected, the closer the metering fix to the aircraft when receiving the CTA, the lower the CTA window. 
Furthermore, it has been also observed that minimum time (i.e. faster) trajectories are very similar to those of minimum fuel, provided that the optimisation takes place once in the descent (the top of descent has been overflown) and neither additional thrust nor drag devices usage are allowed. The reasoning behind this aparent paradox is that minimum time and minimum fuel trajectories select similar altitude profiles, and the idle fuel flow mainly depends on altitude.

It has been also found that there is a trade-off between maximising the robustness of a CDO in the face of late changes to the CTA throughout the descent (i.e. maximising the probability to achieve the whole descent at thrust idle) and minimising the fuel consumption.

In order to maximise the potential benefits of time-based CDO in a future trajectory-based air traffic management paradigm, each aircraft in the merging sequence should provide the ATC with its own feasible CTA window. This will also require advanced on-board real-time trajectory (re)planning systems, capable to support CTA updates during the descent, and advanced ground-based CTA scheduling algorithms.

In future works, the absolute CTA window could be computed allowing the aircraft to apply thrust or speed-brakes if required. The extended CTA window and the associated cost (in terms of additional fuel consumption and noise nuisance) could be analysed. Finally, it would be also interesting to compare the energy-neutral CTA window with that achievable by using path lengthening or stretching, even though pilots would probably prefer energy management to reduce workload and increase situation awareness.

\section{Acknowledgments}

The authors would like to thank Airbus Industries for the use of PEP (Performance Engineers Program) suite, which allowed us to undertake realistic aircraft performances simulations.

\section{References}

[1] Airbus, 1993. Flight Crew Operation Manual (FCOM). A320. Version 1.3.1. Training and flight operations support and services.

[2] Betts, J., 2010. Practical Methods for Optimal Control and Estimation Using Nonlinear Programming, 2nd Edition. Society for Industrial and Applied Mathematics. 
[3] Bronsvoort, J., Mcdonald, G., Lopez-leones, J., Vilaplana, M., Besada, J. A., 2012. Understanding Time-Drift for Different Aircraft Descent Guidance Strategies. In: International Conference on Application and Theory of Automation in Command and Control Systems.

[4] Bryson, A. E., Ho, Y.-C., 1975. "Applied optimal control : optimization, estimation, and control". Taylor and Francis Group, New York, United States of America.

[5] Clarke, J. B., Ho, N. T., Ren, L., Brown, J. A., Elmer, K. R., Zou, K., Hunting, C., McGregor, D. L., Shivashankara, B. N., Tong, K., Warren, A. W., Wat, J. K., Sep. 2004. Continuous descent approach: Design and flight test for Louisville international airport. Journal of Aircraft 41 (5), 1054-1066.

[6] Dalmau, R., Prats, X., Mar 2015. Fuel and time savings by flying continuous cruise climbs: Estimating the benefit pools for maximum range operations. Transportation Research - Part D: Transport and Environment $35,62-71$.

[7] de Boor, C., 2001. A Practical Guide to Splines. Applied Mathematical Sciences. Springer New York.

[8] de Jong, P. M. A., 2014. Continuous descent operations using energy principles. Ph.D. thesis, TUD Technische Universiteit Delft.

[9] de Jong, P. M. A., de Gelder, N., Verhoeven, R. P. M., Bussink, F. J. L., Kohrs, R., van Paassen, M. M., Mulder, M., Nov 2014. Time and energy management during descent and approach: Batch simulation study. Journal of Aircraft 52 (1), 190-203.

[10] Hellmann, G., 1916. Ueber die bewegung der luft in den untersten schichten der atmosphäre. Meteorol Z 34, 273-285.

[11] Hoekstra, J., June 2016. Aircraft performance models for atm research. In: Tutorials at the 7th International Congress on Research in Air Transportation (ICRAT). FAA/EUROCONTROL, Philadelphia (EEUU).

[12] ICAO, 1993. Manual of the ICAO Standard Atmosphere: Extended to 80 Kilometres (262500 Feet). Tech. rep., International Civil Aviation Organization, Montreal, Canada. 
[13] Kaltschmitt, M., Streicher, W., Wiese, A., 2007. Renewable Energy: Technology, Economics and Environment. Springer Berlin Heidelberg.

[14] Kim, B., Li, L., Clarke, J.-P., feb 2014. Runway Assignments That Minimize Terminal Airspace and Airport Surface Emissions. Journal of Guidance, Control, and Dynamics 37 (3), 789-798.

[15] Klooster, J. K., Del Amo, A., Manzi, P., June 2009. Controlled timeof-arrival flight trials. In: 8th USA/Europe Air Traffic Management Research and Development Seminar.

[16] Lindsay, P., Ramsay, C., Vilaplana, M., Leones, J. L., Casado, E., Parks, P., September 2009. Robustness of idle-throttle continuous descent approach trajectories against modified timing requirements. In: 9th AIAA Aviation Technology, Integration, and Operations Conference (ATIO). American Institute of Aeronautics and Astronautics.

[17] Man, L., 2015. An Agent-based Approach to Automated Merge 4D Arrival Trajectories in Busy Terminal Maneuvering Area. Procedia Engineering 99, 233-243.

[18] Park, S. G., Clarke, J., 2013. Feasible time range analysis of wide fleet for continuous descent arrival. In: 2013 Aviation Technology, Integration, and Operations Conference. American Institute of Aeronautics and Astronautics.

[19] Park, S. G., Clarke, J., 2015. Optimal control based vertical trajectory determination for continuous descent arrival procedures. Journal of Aircraft 52 (5), 1469-1480.

[20] Prats, X., Bussink, F., Verhoeven, R., Marsman, A., Sep 2015. Evaluation of in-flight trajectory optimisation with time constraints in a moving base flight simulator. In: Proceedings of the 34th Digital Avionics Systems Conference. IEEE/AIAA, Prague (Czech Republic).

[21] Prats, X., Prez-Batlle, M., Barrado, C., Vilardaga, S., Bas, I., Birling, F., Verhoeven, R., Marsman, A., Jun 2014. Enhancement of a time and energy management algorithm for continuous descent operations. In: Proceedings of the 14th AIAA Aviation Technology, Integration, and Operations Conference, AIAA Aviation and Aeronautics Forum and Exposition. AIAA, Atlanta, Georgia (USA), (AIAA paper 2014-3151). 
[22] Senzig, D. A., Fleming, G. G., June 2009. Fuel consumption modeling in support of atm environmental decision-making. In: 8th USA/Europe Air Traffic Management Research and Development Seminar.

[23] Senzig, D. A., Fleming, G. G., Iovinelli, R. J., Jul. 2009. Modeling of Terminal-Area Airplane Fuel Consumption. Journal of Aircraft 46 (4), 1089-1093.

[24] SESAR Joint Undertaking, July 2015. SESAR Concept of Operations Step 1 Final Edition. Project Deliverable D124, Edition 02.01.00, Brussels.

[25] Takeichi, N., 2017. Nominal flight time optimization for arrival time scheduling through estimation/resolution of delay accumulation. Transportation Research Part C: Emerging Technologies 77, 433 - 443.

[26] Takeichi, N., Inami, D., Nov. 2010. Arrival-Time Controllability of Tailored Arrival Subjected to Flight-Path Constraints. Journal of Aircraft 47 (6), 2021-2029.

[27] Vilardaga, S., Prats, X., 2015. Operating cost sensitivity to required time of arrival commands to ensure separation in optimal aircraft $4 \mathrm{~d}$ trajectories. Transportation Research Part C: Emerging Technologies $61,75-86$. 